\title{
LA FORMACIÓN PROFESIONAL, UNA RESPUESTA EDUCATIVA EFECTIVA PARA ATENDER EL DERECHO AL TRABAJO
}

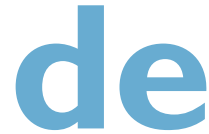

nuestros

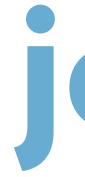

DOI

http://dx.doi.org/pym.i359.y2014.009

Antonio Arenas Alonso

Profesor jubilado del ICAI

Exvicerrector de Ordenación Académica. Universidad Pontificia Comillas aarenas1947@gmail.com

uiero aportar una reflexión personal sobre ciertos equilibrios que debe cuidar nuestro sistema educativo. Equilibrio en el individuo, entre formación generalista y capacidad para el desarrollo de una actividad laboral, y equilibrio entre los niveles de cualificación profesional de la oferta formativa y las demandas de nuestras empresas. Si no, los cuidamos nos encontramos, por un lado, con una excesiva sobrecualificación y, por otro, con una falta de motivación para el estudio que se manifiesta en nuestras elevadas tasas de fracaso escolar, que a su vez tiene incidencia en la actual situación de paro, principalmente juvenil '.

Esta reflexión la realizo tanto desde mi vivencia personal ${ }^{2}$ como desde la experiencia acumulada por el ICAI (Sanz de Diego, 2009) y que a mí tanto me ha aportado en este campo (Arenas et al., 20l3). Así, desde esta posición me atrevo a plantear una línea de pensamiento novedosa y desafiante sobre la estructura de nuestro sistema educativo.

En síntesis, mi tesis principal en este trabajo es muy clara: la Universidad debería acercarse a la Formación Profesional, no sólo para facilitar a los egresados de FP su incorporación a los estudios superiores, sino también para estar más en contacto con los centros docentes generándose confianza y colaboraciones mutuas, que a largo plazo acaben convirtiendo la Formación Profesional en el camino recorrido por la inmensa mayoría de los ciudadanos para alcanzar las

I «Los problemas no resueltos que arrastra el sistema educativo y los desajustes existentes en la interacción entre el sistema educativo y el sistema productivo lastran las posibilidades de desarrollo económico y social. Por ello, mejorar el sistema educativo español y la relación con el sistema productivo es un elemento estratégico para el país y para su desarrollo futuro»» (CES,2009, conclusiones).

2 Un breve historial profesional del autor puede ayudar a situar la reflexión: doctor, ingeniero industrial e ingeniero técnico industrial, (ICAI). Ayudante de ingeniero (ICAI). Maestro industrial. Oficial industrial tornero. Profesor jubilado del Departamento de Ingeniería mecánica de la ETS de Ingeniería ICAI (Universidad Pontificia Comillas). Desarrollando mi actividad profesional como tornero (1962 a 1966), profesor en Escuelas Profesionales Padre Piquer (1966 a 1970) y en la ETS de Ingeniería (ICAI), Universidad Pontificia Comillas (1970 a 2010).
La Universidad debería acercarse a la Formación Profesional, no sólo para facilitar a los egresados de FP su incorporación a los estudios superiores, sino también para estar más en contacto con los centros docentes generándose confianza y colaboraciones mutuas, que a largo plazo acaben convirtiendo la Formación Profesional en el camino recorrido por la inmensa mayoría de los ciudadanos para alcanzar las titulaciones universitarias (máximas cualificaciones profesionales), dejando el camino del Bachillerato para minorías.

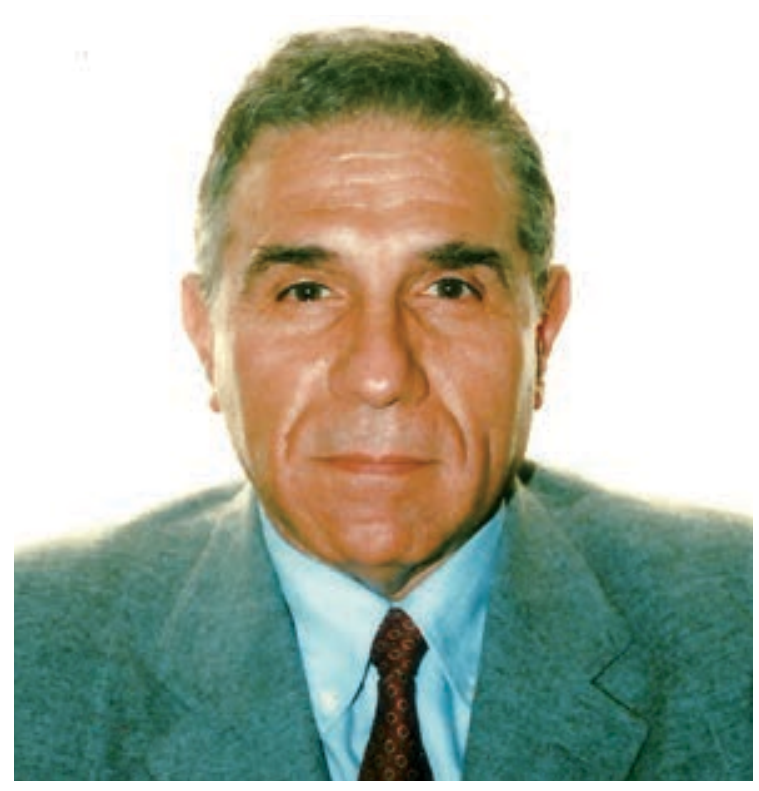

Antonio Arenas Alonso. 
titulacionesuniversitarias (máximascualificaciones profesionales), dejando el camino del Bachillerato para minorías. Ello permitiría que el escalonamiento de estudios fuera acompañado de un escalonamiento profesional, facilitando el acceso al trabajo de los jóvenes y la reducción del fracaso escolar, al mismo tiempo que se produciría una mayor eficacia y eficiencia de los recursos empleados. Se proponen medidas para alcanzar este objetivo de forma progresiva.

\section{El derecho al trabajo y el deber de trabajar}

La situación actual sobre el desempleo juvenil en España nos resulta escandalosa, pero hemos de admitir que no es la primera vez que se producen cifras similares, aunque quizá la percepción social no alcanzó el nivel de gravedad que ahora se empieza a considerar. Una posible diferencia entre el pasado y el momento actual puede ser que en el pasado trabajar era sólo obligación de una parte de la sociedad, y ahora lo entendemos como un derecho y una obligación tanto del individuo como de la sociedad.

Así, no será hasta la constitución de 1978, actualmente en vigor, donde de forma clara y contundente, se reconoce no sólo la obligación, sino también el derecho de los ciudadanos al trabajo ${ }^{3}$. El art. 35 establece que "Todos los españoles tienen el deber de trabajar y el derecho al trabajo, a la libre elección de profesión u oficio, a la promoción a través del trabajo y a una remuneración suficiente para satisfacer sus necesidades y las de su familia, sin que en ningún caso pueda hacerse discriminación por razón del sexo”.

\section{El tRABAJO ES UN VALOR}

En el conjunto de los derechos del ser humano, hay un grupo de ellos que son en mayor o menor grado controlados por los estados (la justicia, la sanidad, la educación), pero hay otros que, aunque proclamados por los estados (la vivienda, fundar una familia, fijación de residencia, el trabajo...), quedan libres y están muy condicionados por la situación socioeconómica. Entre estos, el trabajo es la llave, pues será mediante el trabajo como se conseguirán los medios económicos y la autonomía personal real que permita acceder a ellos. Más aún, en la economía de mercado en la que vivimos, el dinero es el medio básico para obtener los bienes y servicios necesarios, y la forma fundamental y socialmente más valorada para conseguir éste dinero es el trabajo.

Me atrevo a decir que la capacidad para el desarrollo de un trabajo se encuentra entre los que consideramos valores de la persona. Así, ipuede decirse en nuestra sociedad que una persona está plenamente formada si no cuenta con la capacidad para el desarrollo de una actividad laboral? En consonancia, hoy día

3 En el resto de nuestras anteriores normas constitucionales, no aparecen sino referencias a la obligación de los ciudadanos a contribuir económicamente a los gastos del estado (Constitución de I8I2, art. 8; Constitución de 1837, art. 6; Constitución de 1845, art. 6) o a la libertad de elección de profesión (Constitución de 1876, art. 12). ise puede plantear el sistema educativo de un país sin que entre sus objetivos se encuentre conseguir personas con capacidad para el desarrollo de un trabajo?

Así, el sistema educativo habría de ser tal que lo cotidiano y normal sea que, al alcanzar la mayoría de edad, los ciudadanos hayan alcanzado junto a su formación en valores ciudadanos las capacidades laborales necesarias que les permitan ejercer su derecho al trabajo. A partir de aquí, el ciudadano en el uso de su libertad y atendiendo a múltiples condicionantes personales y sociales sería quien decidiría aplicar estas capacidades (es decir, incorporarse al mundo del trabajo) o posponerlo, avanzando en la consecución de mayores niveles de formación, tanto en valores ciudadanos como profesionales. El resultado sería un crecimiento escalonado y simultáneo en valores ciudadanos y capacidades profesionales, cerrando ciclos académicos completos. Es decir, en mi opinión, salvo personas excepcionales y en profesiones excepcionales, no se debería alcanzar la máxima cualificación en una profesión (formación universitaria) sin haber obtenido cualificaciones inferiores; esto aseguraría una mayor madurez en la persona y en el conocimiento de las materias, y redundaría claramente tanto en una mayor eficiencia y eficacia de nuestro sistema educativo como en la satisfacción final de sus usuarios con el mismo.

Una estructura educativa que permita el avance en la formación de profesionales apoyándose en escalones con cualificaciones a partir de las ya obtenidas permite optimizar recursos y reducir fracasos, pues:

a) Facilita la entrada y salida del mundo académico según las capacidades, las aptitudes, las necesidades y la situación social de cada persona.

b) Favorece que la progresión hacia niveles académicos superiores se vaya produciendo en función de dichas capacidades, aptitudes y necesidades, contribuyendo con ello desde el sistema educativo y de forma natural, a obtener una estructura piramidal de profesionales acorde con las necesidades del sistema productivo.

\section{Nuestro sistema educativo}

Planteado así el trabajo como derecho y obligación, quiero acercarme a la relación entre formación y trabajo en España, es decir, quiero analizar cómo se enfoca en nuestro sistema educativo la preparación de la persona para que pueda desarrollar este derecho tan fundamental, cuando alcanza la mayoría de edad.

En este contexto, y por motivos de claridad, en adelante llamaremos "estudios profesionales" a aquellos estudios que tienen entre sus objetivos el desarrollo de capacidades para realizar una actividad laboral, y "estudios generalistas" a aquellos que no cuentan entre sus objetivos el alcanzar dichas capacidades. Así, en nuestro sistema educativo encuadraríamos en el se- 
Figura I. Itinerarios de enseñanza en el sistema educativo español, eXcluidas las enseñanzas ESPECIALES (MÚSICA, DANZA, DEPORTIVAS, ARTES PLÁSTICAS Y DISEÑO)

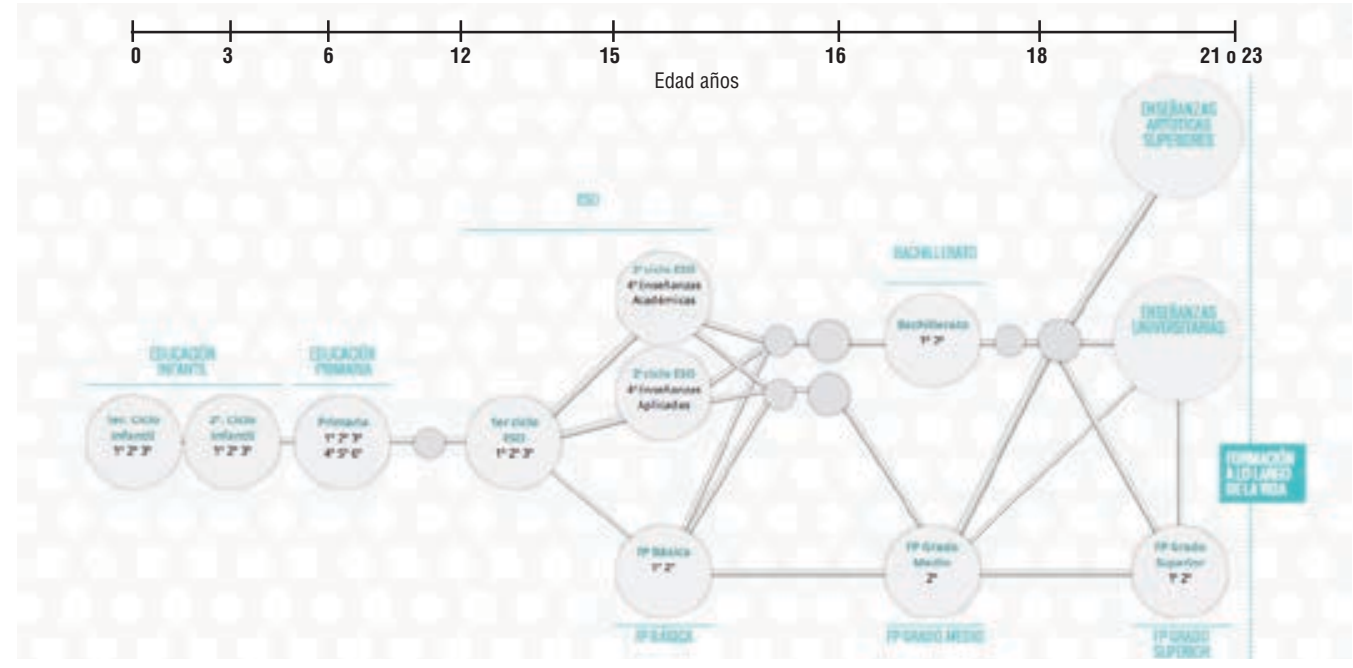

Fuente: MEC (http://lomce.mecd.es/itinerarios).

gundo grupo a la ESO y al Bachillerato, $y$ en el primer grupo a la Formación Profesional y a las carreras universitarias ${ }^{4}$.

De este modo, el reciente cambio legal (LOMCE, 2013) en nuestro sistema educativo plantea en su concepción básica (ver Figura I) un tronco común de enseñanza obligatoria que cubre el periodo hasta los quince años, edad en la que se plantea la opción de $2^{\circ}$ ciclo de ESO (estudios generalistas) o FP básica. A partir de este momento el sistema se divide en dos caminos aparentemente paralelos con pasarelas entre ellos. El camino generalista comprende el $2^{\circ}$ ciclo de ESO y el Bachillerato, y el camino profesional, la FP Básica y la FP de grado medio.

Así, al alcanzar los 18 años ${ }^{5}$ únicamente tendrán capacidad para el desarrollo de una actividad laboral quienes al finalizar la ESO hayan elegido la FP de grado medio, no así quienes hayan realizado Bachillerato ${ }^{6}$. Al finalizar el Bachillerato, de nuevo se plantea para la etapa de la Educación Superior los dos caminos alternativos, acceder a la vía de la Formación Profesional o la de los grados universitarios ${ }^{7}$.

4 Nótese que la terminología utilizada en nuestro sistema es ambigua, pues no sólo se forman profesionales a través de la llamada Formación Profesional, también se forman profesionales a través de la formación universitaria. Sin embargo a la formación en la universidad no se le aplica el nombre de profesional, aunque en la sociedad a los titulados universitarios sí se les reconoce y denomina como profesionales en el sector correspondiente.

5 Supuesta una progresión normal en el desarrollo de los estudios anteriores, y por tanto que no se ha producido repetición de curso.

6 Es evidente que la idea que subyace en este planteamiento es que, en la sociedad actual, sin formación específica es muy difícil y a veces imposible el desarrollo de una actividad laboral. El elevado nivel de tecnificación de nuestra sociedad normalmente requiere una elevada especialización para los puestos de trabajo.

7 En realidad el camino de los estudios universitarios también es un camino de profesionalización, pues ciertamente la inmensa mayoría de los jóvenes buscan con estos estudios una salida profe-
Por consiguiente, quienes siguen el camino generalista hasta el final, únicamente adquirirían capacidad para el desarrollo de una actividad laboral cuando finalicen sus estudios universitarios, hecho que sucederá como mínimo a los veintiún años. Esta circunstancia no parece revestir ninguna preocupación si se considera de forma aislada, pero a mi parecer es tremendamente grave cuando se pone en el contexto de las siguientes consideraciones:

- En el diseño de nuestro sistema educativo la Formación Profesional se configura como un camino secundario alternativo para los que fracasan en el primer camino. Esta premisa que puede ser inicialmente negada por algunos, sin embargo está plenamente demostrada cuando se analiza con detenimiento nuestra legislación educativa, no sólo la LOMCE, sino todas las leyes desde el siglo XVIII.

- Lo anterior significa que nuestro sistema educativo establece el camino generalista como camino principal, es decir, que si no hubiera fracaso escolar, nuestros alumnos serían todos universitarios, y así la primera capacitación para el ejercicio de una actividad laboral sería del máximo nivel. Esto generaría una estructura de oferta profesional cilíndrica, frente a la habitual demanda empresarial piramidal, en la que a medida que crece el nivel de la cualificación profesional demandado, decrece el número de puestos de trabajo, (Arenas 2009), produciéndose sobrecualificación (CES, 2009), con un claro desajuste generador de frustraciones $y$ de gasto inútil.

sional. Esto nos lleva a observar que en realidad hay una formación profesional de primera (la universitaria) y otra de segunda, la llamada Formación Profesional. 
- Dado que existe fracaso académico ${ }^{8}$, la salida diseñada para quienes no pueden seguir el camino generalista es la Formación Profesional, convirtiéndose así éste en camino de fracasados?

- La realidad del fracaso académico nos lleva a que la salida de la universidad para desarrollar la primera actividad laboral no sea a los veintiún años, sino a edades muy superiores, a veces cercanas a los treinta años ${ }^{10}$.

- También la realidad del fracaso académico nos conduce al abandono de los estudios iniciados (casi siempre en el camino generalista) y a la incorporación al mundo de trabajo o al camino profesional, con edades inapropiadas y con un alto grado de desmotivación y desánimo.

- Con o sin fracaso académico, los costes de la sobrecualificación de profesionales, del retorno al camino profesional y del abandono de procesos formativos sin concluirlos para incorporarse al mundo del trabajo, suponen cifras que no son ni justificables ni aceptables en nuestra sociedad.

\section{CONCLUSIONES Y PROPUESTAS}

Al analizar nuestro sistema educativo desde la óptica de la preparación de los ciudadanos para el desarrollo de una actividad laboral, nos encontramos con graves problemas estructurales, no sólo relacionados con el diseño del sistema, sino también de la aplicación del mismo y sobre todo de la percepción social que se tiene de los dos caminos que se configuran.

La esencia del problema radica en que el camino diseñado como principal (camino generalista) y que es el socialmente más demandado (camino de mayorías), es un camino que conduce a cualificaciones profesionales de alto nivel (universitario) sin escalones profesionales intermedios y con muy pocas garantía de inserción laboral en la profesión estudiada en muchas de las titulaciones que la Universidad ofrece. A su vez, el camino que produce cualificaciones de bajo, medio e incluso de alto nivel y escalonado (camino profesional), es un camino diseñado como secundario y poco valorado socialmente, resultando así ser un camino de minorías principalmente provenientes de las bolsas de fracaso escolar que el propio sistema genera en el primer camino.

Tras las reflexiones anteriores, la situación ideal sería que la gran mayoría de nuestros estudiantes se incorporara en el camino profesional como itinerario prin-

8 Posiblemente en mayor número del aceptable por circular por el camino generalista, camino árido y quizá poco amigable por su planteamiento alejado de la realidad laboral.

9 Fracasados no sólo en el camino de la ESO, el Bachiller y la Universidad, sino en el mundo profesional, dado que cada vez se da más el caso de titulados universitarios que se matriculan en cursos de FP. Este hecho revela la grave enfermedad de nuestro sistema, malgastando recursos e ilusiones.

10 El desarrollo de la primera actividad laboral con edades altas implica serios problemas de adaptación, toda vez que los trabajos iniciales suelen demandar aptitudes poco compatibles con edades elevadas. cipal en la educación postobligatoria y únicamente una minoría tuviera acceso al camino generalista, invirtiendo así los flujos actuales.

Consciente de las grandes dificultades existentes en nuestro país para cambios en profundidad y duraderos de nuestro sistema educativo, ni pido ni espero acciones a corto o medio plazo, pues entiendo la gran complejidad del problema. Sin embargo, quiero apuntar una dirección de trabajo que iniciada con cierta premura supondría avances gigantescos en la resolución de los problemas planteados, sin grandes convulsiones en el propio sistema.

Al ser la Universidad la institución que genera más valor añadido a las actividades de formación y mayor reconocimiento a las cualificaciones profesionales, su posicionamiento frente a los dos caminos de posible procedencia de sus alumnos resulta decisivo para la valoración que la sociedad haga de dichos caminos.

Así, la primera propuesta consiste en que la Universidad reconozca a los titulados en Formación Profesional superior que accedan a estudio de grado, un número de créditos igual a los cursados en Formación Profesional. Ello significaría que los alumnos que realizan un grado directamente desde el Bachiller o a través de la Formación Profesional Superior podrían finalizar con la misma edad o, dicho de otra manera, que el tiempo dedicado a la Formación Profesional superior más el que le reste para finalizar el grado sumen igual que el tiempo que dedicaría un alumno de Bachiller para obtener el grado.

Para que esta propuesta pueda ser realista y efectiva, es necesario que las universidades conozcan bien los programas de los estudios de Formación Profesional superior y reconozcan su calidad, así como que los centros de formación profesional generen en aquellas la suficiente confianza. Así, se sugiere que las universidades establezcan acuerdos de colaboración con uno o varios centros de formación profesional, creándose un campus profesional configurado por la Universidad, los centros de Formación Profesional y las empresas colaboradoras de éstos, en el que se desarrolle un trabajo cooperativo con mutua confianza. Entre otros acuerdos, en el orden académico se apuntan los siguientes:

- La Universidad participaría en la elaboración de los programas de contenidos concretos en los estudios de Formación Profesional superior.

- La Universidad participaría en la elaboración y supervisión de las pruebas de evaluación conducentes a la obtención de los títulos de Formación Profesional Superior.

- Profesores de la Universidad podrían impartir docencia de las materias acordadas en el centro de formación profesional.

- La Universidad podría participar en la elaboración de materiales docentes utilizados en las enseñanzas de Formación Profesional superior. 


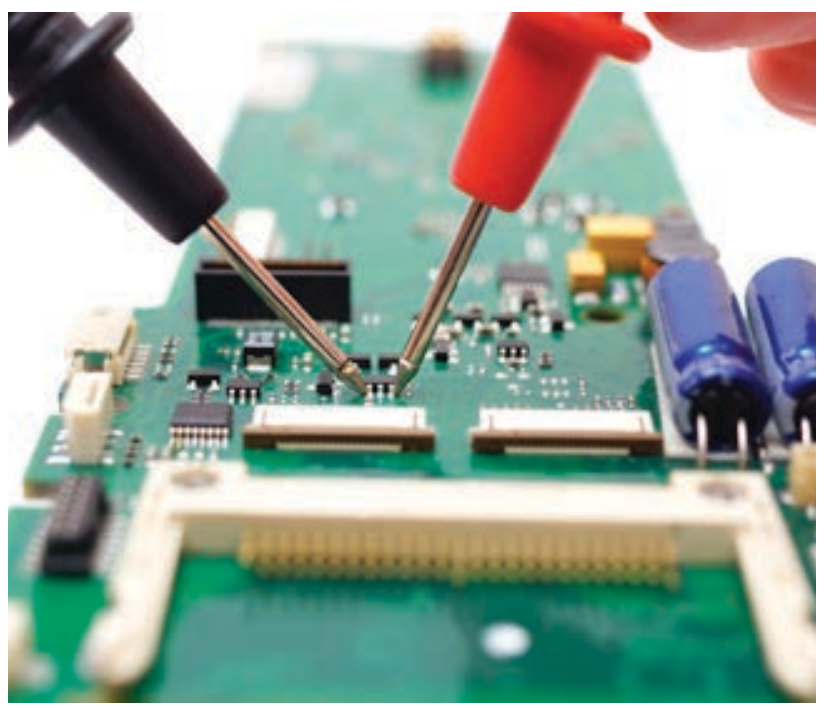

- La Universidad y el centro de Formación Profesional podrían compartir aulas, laboratorios y talleres, en orden a una optimización y complementariedad de los recursos.

- El centro de Formación Profesional participaría en la definición y aplicación de los requisitos de acceso a los estudios de grado, en caso de existir dichos requisitos.

De este campus profesional saldrían grandes ventajas para todos los implicados. La universidad contaría con canteras de futuros alumnos con conocimiento de su trayectoria. Los centros de formación profesional generarían un atractivo muy importante al ofrecer a sus egresados la posibilidad de continuar estudios universitarios con el pleno reconocimiento de los conocimientos y valores adquiridos. Las empresas, ya colaboradoras de los centros de Formación Profesional, podrían ampliar sus relaciones y posibilidades de colaboración con la universidad [Arenas, 2010].

La segunda propuesta sería de fácil implementación una vez superada la primera, consiste en que la Universidad otorgue prioridad para el acceso a sus estudios a los alumnos provenientes de la Formación Profesional superior. Es necesario para ello que las universidades rediseñen sus planes de estudio alineados congruentemente con los títulos que se ofrecen en la Formación Profesional superior y con las demandas reales del mercado laboral de titulados universitarios.

\section{Para saber más}

- Arenas et al. (2013): “TCA. Una experiencia de enseñanza universitaria en alternancia en el ICAI de los años 90". Anales de Mecánica y Electricidad, noviembre-diciembre 2013.

- Arenas (2009): "Reflexiones sobre la dignificación de la Formación Profesional. (Carta abierta al Sr. Ministro de Educación)". Anales de Mecánica y Electricidad, noviembre-diciembre 2009.

- Arenas (2010): “La Formación Profesional en el Pacto por la Educación”. Anales de Mecánica y Electricidad, mayo-junio 2010.

- CES 2009. Sistema educativo y capital humano. Informe 01/2009 del Consejo Económico y Social de España.

- Constitución española, 1978.

- Ley Orgánica de educación, 2006.

- Ley Orgánica para la Mejora de la Calidad Educativa, LOMCE, 2013.

- Sanz de Diego, R. (2009): ICAI 1908-2008. Lo que fuimos, lo que somos. Madrid: Universidad Pontificia Comillas.

\section{hemos hablado de:}

\section{Formación profesional; colaboración FP y} empresa; calidad de la FP; derecho al trabajo.

Este artículo fue solicitado por PADRES Y MAESTROS en junio de 2014, revisado y aceptado en septiembre de 2014 para su publicación.

\section{Próximos números}

\section{Diciembre}

\section{ACCIÓN TUTORIAL CON LAS FAMILIAS}

¿Cómo crear sinergias con las familias en la era del aprendizaje?

La labor tutorial con las familias

La acción tutorial con familias de alumnos con necesidades educativas especiales

Escuela y familia $\mathbf{2 . 0}$

Inteligencias múltiples. Coordenadas para una colaboración efectiva familia-centro

\section{SECCIONES}

Filmoteca Padres y Maestros

Experiencias de innovación

Una mirada sobre la educación

Grandes de la educación

Orientación Educativa

Biblioteca PyM

Febrero

LIDERAZGO PEDAGÓGICO 